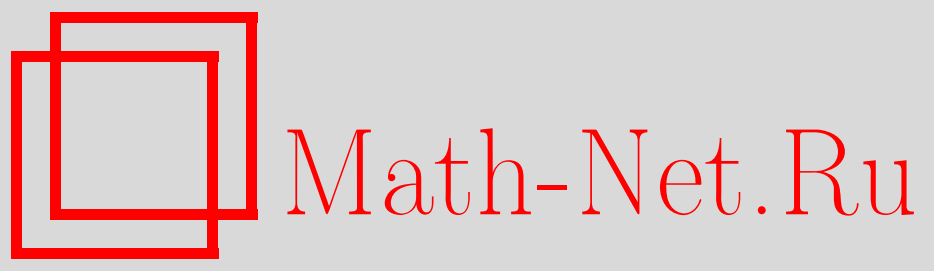

Н. В. Алексеева, Е. В. Земляная, Бризеры в нелинейном уравнении Шредингера с накачкой и диссипацией, ТМФ, 2011, том 168, номер 1, 4-12

DOI: https://doi.org/10.4213/tmf6659

Использование Общероссийского математического портала Math-Net.Ru подразумевает, что вы прочитали и согласны с пользовательским соглашением http://www.mathnet.ru/rus/agreement

Параметры загрузки:

IP: 54.197 .130 .99

26 апреля 2023 г., 15:36:36

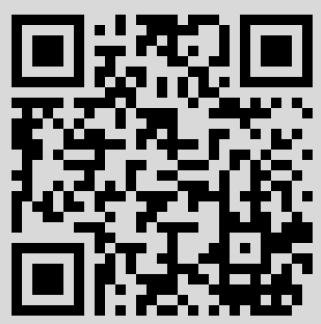




\section{БРИЗЕРЫ В НЕЛИНЕЙНОМ УРАВНЕНИИ ШРЕДИНГЕРА С НАКАЧКОЙ И ДИССИПАЦИЕЙ}

Периодические во времени солитоны нелинейного уравнения Шредингера с диссипацией и параметрической накачкой строятся как решения граничной задачи для пространственно-зависимых коэффициентов Фурье.

Ключевые слова: нелинейное уравнение Шредингера, устойчивость, солитоны, диссипация, параметрическая накачка, бифуркации.

\section{1. ВВЕДЕНИЕ}

Нелинейное уравнение Шредингера с диссипацией и параметрической накачкой

$$
i \psi_{t}+\psi_{x x}+2|\psi|^{2} \psi-\psi=h \psi^{*}-i \gamma \psi
$$

где $\gamma>0$ - коэффициент затухания, а $h>0$ - амплитуда параметрической накачки, описывает ряд резонансных явлений в нелинейных средах - таких, например, как нелинейный фарадеев резонанс в вертикально осциллирующем водяном желобе [1] и эффект фазового усиления солитонов в оптическом волокне [2]. Это же уравнение используется при описании волн намагниченности в ферромагнетике типа легкая плоскость, подверженном комбинированному воздействию статического и микроволнового полей [3], и при расчете амплитуды синхронизованных колебаний в вертикально раскачиваемых маятниковых решетках [4].

Нелинейное уравнение Шредингера с накачкой и диссипацией поддерживает локализованные решения с различными типами зависимости от времени. Два сосуществующих стащионарных солитона, обозначаемых через $\psi_{+}$и $\psi_{-}$, определяются следующими выражениями:

$$
\psi_{ \pm}(x)=A_{ \pm} e^{-i \theta_{ \pm}} \operatorname{sech}\left(A_{ \pm} x\right)
$$

где

$$
A_{ \pm}=\sqrt{1 \pm \sqrt{h^{2}-\gamma^{2}}}, \quad \theta_{+}=\frac{1}{2} \arcsin \frac{\gamma}{h}, \quad \theta_{-}=\frac{\pi}{2}-\theta_{+}
$$

*Department of Mathematics, University of Cape Town, Cape Town, South Africa. E-mail: nora.alexeeva@uct.ac.za

†Объединенный институт ядерных исследований, Дубна, Московская обл., Россия. E-mail: elena@jinr.ru 
Солитон $\psi_{-}$неустойчив при всех $h$ и $\gamma$, тогда как $\psi_{+}$является устойчивым при $h<h_{\mathrm{H}}(\gamma)$ и неустойчивым в противном случае [3]. Значение $h_{\mathrm{H}}(\gamma)$ лежит между $h=\gamma$ (нижняя граница области существования обоих солитонов) и $h=\sqrt{1+\gamma^{2}}$ (точка, в которой тривиальное решение $\psi=0$ теряет устойчивость). Если $h$ растет при фиксированном значении $\gamma$ и становится больше $h_{\mathrm{H}}(\gamma)$, то решение $\psi_{+}$претерпевает бифуркацию Хопфа [3] и порождает устойчивый периодический во времени солитон, или бризер [5], [6]. При дальнейшем увеличении интенсивности накачки периодический солитон может претерпеть каскад удвоений периода или уступить место какому-то нелокализованному аттрактору в зависимости от значения $\gamma$ [5].

Существование, устойчивость и бифуркации стационарных солитонов могут быть исследованы с помощью различных аналитических и численных методов. К сожалению, бо́льшая часть этих методов неприменима к периодическим по времени солитонам, для которых непосредственное численное моделирование является наиболее эффективным методом анализа. Недостатком этого метода является, однако, то, что моделирование возможно только в случае устойчивых солитонов. Это означает, что как сами механизмы, так и нюансы взаимопревращений солитонов (которыми являются бифуркации с участием устойчивых и неустойчивых решений) остаются недоступными для изучения. Неприменимо моделирование и для нахождения сосуществующих аттракторов в случаях би- или мультистабильности.

Альтернативным подходом является изучение периодических солитонов как решений граничной задачи, сформулированной в двумерной (цилиндрической) пространственно-временной области [7]-[9]. Преимущество такого подхода состоит в том, что он дает как устойчивые, так и неустойчивые решения. Его основным недостатком является потребность в значительных вычислительных ресурсах (компьютерной памяти и времени работы процессора), необходимых для достижения разумной точности вычислений. Эти ресурсы быстро исчерпываются, если расширять двумерную область по одному из двух измерений либо увеличивать количество точек дискретной сетки или число фурье-гармоник. В результате оказывается невозможно получить солитоны с большими временны́ми периодами или медленным затуханием по пространственным координатам. В итоге попытки найти численное решение с более высокой точностью упираются в непреодолимые ограничения по ресурсам.

В настоящей статье мы обходим эти трудности, предлагая недорогой с вычислительной точки зрения приближенный метод нахождения пространственно-локализованных периодических по времени решений. Дифференциальное уравнение в частных производных сводится к системе связанных обыкновенных дифференциальных уравнений, которые затем решаются численно в одномерной области. Мы показываем, как бифуркации бризеров переходят в бифуркации решений этой редуцированной системы и, в частности, как в рамках этой системы идентифицировать бифуркации удвоения периода.

\section{2. ПРОЦЕДУРА СВЕДЕНИЯ}

С помощью разбиения

$$
\psi=A_{+} e^{-i \theta_{+}}[U(\tilde{x}, \tilde{t})+i V(\tilde{x}, \tilde{t})]
$$


где $A_{+}$и $\theta_{+}$те же, что и в соотношениях (3), и $\tilde{t}=A_{+}^{2} t, \tilde{x}=A_{+} x$, уравнение (1) переписывается в виде

$$
\begin{gathered}
-V_{t}-2 \Gamma V=-U_{x x}+U-2\left(U^{2}+V^{2}\right) U \\
U_{t}+2 H V=-V_{x x}+V-2\left(U^{2}+V^{2}\right) V
\end{gathered}
$$

(здесь и далее мы опускаем знак тильды над $x$ и $t$.) В последних уравнениях мы ввели $\Gamma=\gamma / A_{+}^{2}$ и $H=\sqrt{h^{2}-\gamma^{2}} / A_{+}^{2}$.

Периодические функции $U(x, t)$ и $V(x, t)$ могут быть разложены в ряды Фурье

$$
U=\sum_{n=-N}^{N} U_{n} e^{i n \Omega t}+\text { к. с., } \quad V=\sum_{n=-N}^{N} V_{n} e^{i n \Omega t}+\text { к. с. },
$$

где $N=\infty$. В данных соотношениях $\Omega=2 \pi / A_{+}^{2} T$, где $T-$ период решения уравнения (1). При приближении к точке бифуркации Хопфа коэффициенты Фурье $U_{n}$ и $V_{n}$ убывают как $\epsilon^{|n|}[6]$. Численный анализ указывает, что этот степенной закон остается в силе в широком диапазоне изменения параметров. Таким образом, если $\epsilon$ мало, то гармоники более высокого порядка имеют пренебрежимо малые амплитуды, и ряд может быть оборван на некотором конечном $N$.

Самое грубое приближение состоит в удержании лишь нулевой и первой гармоник (т. е. в разложении (6) полагаем $N=1$ ). Чтобы упростить обозначения, мы переобозначим амплитуды двух мод следующим образом:

$$
\begin{aligned}
& U(x, t)=u(x)+\mathcal{A}(x) e^{i \Omega t}+\mathcal{A}^{*}(x) e^{-i \Omega t}, \\
& V(x, t)=v(x)+\mathcal{B}(x) e^{i \Omega t}+\mathcal{B}^{*}(x) e^{-i \Omega t},
\end{aligned}
$$

где $u$ и $v$ - вещественные функции, а $\mathcal{A}$ и $\mathcal{B}$ - комплексные коэффициентные функции, стремящиеся к нулю при $|x| \rightarrow \infty$. Подстановка этих выражений в уравнения (5) и приравнивание коэффициентов при одинаковых гармониках дает

$$
\begin{aligned}
u_{x x}-u+2\left(u^{2}+v^{2}\right) u+4\left(3|\mathcal{A}|^{2}+|\mathcal{B}|^{2}\right) u+4\left(\mathcal{A B} \mathcal{B}^{*}+\mathcal{A}^{*} \mathcal{B}\right) v-2 \Gamma v & =0 \\
v_{x x}-v+2\left(u^{2}+v^{2}\right) v+4\left(|\mathcal{A}|^{2}+3|\mathcal{B}|^{2}\right) v+4\left(\mathcal{A} \mathcal{B}^{*}+\mathcal{A}^{*} \mathcal{B}\right) u+2 H v & =0 \\
\mathcal{A}_{x x}-\mathcal{A}+2\left(3 u^{2}+v^{2}\right) \mathcal{A}+2\left(3|\mathcal{A}|^{2}+2|\mathcal{B}|^{2}\right) \mathcal{A}+2\left(2 u v+\mathcal{A}^{*} \mathcal{B}\right) \mathcal{B}-2 \Gamma \mathcal{B}-i \Omega \mathcal{B} & =0 \\
\mathcal{B}_{x x}-\mathcal{B}+2\left(u^{2}+3 v^{2}\right) \mathcal{B}+2\left(2|\mathcal{A}|^{2}+3|\mathcal{B}|^{2}\right) \mathcal{B}+2\left(2 u v+\mathcal{B}^{*} \mathcal{A}\right) \mathcal{A}+2 H \mathcal{B}+i \Omega \mathcal{A} & =0 .
\end{aligned}
$$

\section{3. БИФУРКАЦИЯ ХОПФА КАК БИФУРКАЦИЯ ТИПА "ВИЛКА" В РЕДУЦИРОВАННЫХ УРАВНЕНИЯХ}

Одно гомоклиническое решение системы (7) существует при всех $H$ и Г: это $u=\operatorname{sech} x, v=\mathcal{A}=\mathcal{B}=0$. Указанное решение соответствует стационарному солитону $\psi_{+}(x)$ в уравнении (1). Нетривиальное гомоклиническое решение (соответствующее периодически осциллирующему солитону в уравнении (1)) отщепляется от него, если увеличивать $H$ при фиксированном Г. Покажем, что эта бифуркация типа "вилка" происходит строго в точке бифуркации Хопфа стационарного солитона уравнения (1). Наш анализ можно также рассматривать как косвенное подтверждение приведенного выше степенного закона. 
Пусть $H_{0}$ - значение параметра $H$, при котором происходит бифуркация Хопфа; очевидно, $H_{0}=H_{0}(\Gamma)$. Для $H$, близких к $H_{0}$, можно записать $H=H_{0}+\epsilon^{2} H_{2}$, где $\epsilon$ - малый параметр. Предположим, что частота $\Omega$ разлагается как $\Omega=\Omega_{0}+\epsilon^{2} \Omega_{2}+$ $\epsilon^{4} \Omega_{4}+\cdots$, а поля $u(x), v(x), \mathcal{A}(x)$ и $\mathcal{B}(x)$ - как

$$
\begin{aligned}
u & =u_{0}+\epsilon^{2} u_{2}+\epsilon^{4} u_{4}+\cdots, & v & =\epsilon^{2} v_{2}+\epsilon^{4} v_{4}+\cdots \\
\mathcal{A} & =\epsilon \mathcal{A}_{1}+\epsilon^{3} \mathcal{A}_{3}+\cdots, & \mathcal{B} & =\epsilon \mathcal{B}_{1}+\epsilon^{3} \mathcal{B}_{3}+\cdots
\end{aligned}
$$

Подставляя эти разложения в уравнения (7) и приравнивая коэффициенты при $\epsilon^{1}$, получаем

$$
\mathfrak{L}_{\Omega_{0}}\left(\begin{array}{l}
\mathcal{A}_{1} \\
\mathcal{B}_{1}
\end{array}\right) \equiv\left(\begin{array}{cc}
L_{1} & \Gamma+i \Omega_{0} \\
-i \Omega_{0} & L_{0}-2 H_{0}
\end{array}\right)\left(\begin{array}{l}
\mathcal{A}_{1} \\
\mathcal{B}_{1}
\end{array}\right)=0
$$

где использованы стандартные обозначения для операторов Штурма-Лиувилля $L_{0}=-d^{2} / d x^{2}+1-2 u_{0}^{2}$ и $L_{1}=-d^{2} / d x^{2}+1-6 u_{0}^{2}$. Значения $H_{0}$ и $\Omega_{0}$ выбираются так, что оператор в левой части уравнения (8) имеет нулевое собственное значение, т. е. уравнение $(8)$ имеет нетривиальное решение $\left(\mathcal{A}_{1}, \mathcal{B}_{1}\right)$. Оператор $\mathfrak{L}_{\Omega_{0}}$ совпадает с линеаризованным оператором уравнения (1) в частных производных; именно это соответствие гарантирует совпадение точек бифуркации уравнений (1) и (7).

Указанный собственный вектор можно записать следующим образом:

$$
\left(\begin{array}{l}
\mathcal{A}_{1} \\
\mathcal{B}_{1}
\end{array}\right)=\left(\begin{array}{c}
\mathcal{A}_{0} \\
z^{-1} \mathcal{B}_{0}
\end{array}\right)
$$

где $\left(\mathcal{A}_{0}, \mathcal{B}_{0}\right)^{\mathrm{T}}$ является собственным вектором, отвечающим нулевому собственному значению для оператора $\mathfrak{L}_{\omega}$ с $\Gamma=0$ и комплексным $\omega=z \Omega_{0}$,

$$
\left(\begin{array}{cc}
L_{1} & i \omega \\
-i \omega & L_{0}-2 H_{0}
\end{array}\right)\left(\begin{array}{l}
\mathcal{A}_{0} \\
\mathcal{B}_{0}
\end{array}\right)=0
$$

Здесь $z^{2}=1-2 i \Gamma / \Omega_{0}$. Собственный вектор $\left(\mathcal{A}_{0}, \mathcal{B}_{0}\right)^{\mathrm{T}}$ предполагается нормированным, например $\int\left(\left|\mathcal{A}_{0}\right|^{2}+\left|\mathcal{B}_{0}\right|^{2}\right) d x=1$. Заметим, что $\mathcal{A}_{0}(x), \mathcal{B}_{0}(x)$ и, следовательно, $\mathcal{A}_{1}(x)$ и $\mathcal{B}_{1}(x)$ - четные функции.

Далее, собирая коэффициенты при $\epsilon^{2}$, имеем

$$
\mathfrak{L}_{0}\left(\begin{array}{l}
u_{2} \\
v_{2}
\end{array}\right) \equiv\left(\begin{array}{cc}
L_{1} & 2 \Gamma \\
0 & L_{0}-2 H_{0}
\end{array}\right)\left(\begin{array}{l}
u_{2} \\
v_{2}
\end{array}\right)=4 u_{0}\left(\begin{array}{c}
3\left|\mathcal{A}_{1}\right|^{2}+\left|\mathcal{B}_{1}\right|^{2} \\
\mathcal{A}_{1} \mathcal{B}_{1}^{*}+\mathcal{A}_{1}^{*} \mathcal{B}_{1}
\end{array}\right)
$$

Оператор $\mathfrak{L}_{0}$ имеет единственное нулевое собственное значение с собственным вектором $\left(\partial_{x} u_{0}, 0\right)^{\mathrm{T}}$, следовательно, эрмитово-сопряженный оператор $\mathfrak{L}_{0}^{\dagger}$ также имеет единственное нулевое собственное значение. Легко показать, что связанный с ним собственный вектор имеет вид $\left(\partial_{x} u_{0}, v_{*}\right)^{\mathrm{T}}$, где нечетная функция $v_{*}(x)$ удовлетво- 
ряет уравнению $\left(L_{0}-2 H_{0}\right) v_{*}=-2 \Gamma \partial_{x} u_{0}$. Так как $\mathcal{A}_{1}(x)$ и $\mathcal{B}_{1}(x)$ являются четными функциями, условие разрешимости

$$
\int u_{0}\left(\partial_{x} u_{0}, v_{*}\right)\left(\begin{array}{c}
3\left|\mathcal{A}_{1}\right|^{2}+\left|\mathcal{B}_{1}\right|^{2} \\
\mathcal{A}_{1} \mathcal{B}_{1}^{*}+\mathcal{A}_{1}^{*} \mathcal{B}_{1}
\end{array}\right) d x=0
$$

выполнено, и уравнение (10) допускает решение, стремящееся к нулю при $|x| \rightarrow \infty$.

Переходя к членам порядка $\epsilon^{3}$, получаем уравнение для $\mathcal{A}_{3}$ и $\mathcal{B}_{3}$ :

$$
\mathfrak{L}_{\Omega_{0}}\left(\begin{array}{c}
\mathcal{A}_{3} \\
\mathcal{B}_{3}
\end{array}\right)=\left(\begin{array}{c}
F_{3} \\
G_{3}
\end{array}\right)
$$

где

$$
\begin{aligned}
& F_{3}=\left(12 u_{0} u_{2}+6\left|\mathcal{A}_{1}\right|^{2}+4\left|\mathcal{B}_{1}\right|^{2}\right) \mathcal{A}_{1}+\left(4 u_{0} v_{2}+2 \mathcal{A}_{1}^{*} \mathcal{B}_{1}-i \Omega_{2}\right) \mathcal{B}_{1} \\
& G_{3}=\left(4 u_{0} v_{2}+2 \mathcal{A}_{1} \mathcal{B}_{1}^{*}+i \Omega_{2}\right) \mathcal{A}_{1}+\left(4 u_{0} u_{2}+4\left|\mathcal{A}_{1}\right|^{2}+6\left|\mathcal{B}_{1}\right|^{2}+2 H_{2}\right) \mathcal{B}_{1}
\end{aligned}
$$

Собственным вектором, отвечающим нулевому собственному значению для оператора $\mathfrak{L}_{\Omega_{0}}^{\dagger}$ является $\left(\mathcal{A}_{0}^{*},-z^{*} \mathcal{B}_{0}^{*}\right)^{\mathrm{T}}$, где $\mathcal{A}_{0}$ и $\mathcal{B}_{0}$ определяются уравнением $(9)$. Следовательно, условие разрешимости уравнения (11) записывается как

$$
\int\left(\mathcal{A}_{0}^{*},-z^{*} \mathcal{B}_{0}^{*}\right)\left(\begin{array}{c}
c F_{3} \\
G_{3}
\end{array}\right) d x=0 .
$$

Необходимо выбрать $H_{2}$ и $\Omega_{2}$ так, чтобы действительная и мнимая части интеграла в соотношении (12) были равны нулю. Это обеспечивает существование у уравнения (11) решения, стремящегося к нулю на бесконечности. Для данного $H$ коэффициент $H_{2}$ не определен единственным образом и может быть выбран произвольно; выбор $H_{2}$ просто задает значение $\epsilon$. В отличие от $H_{2}$, полученное значение $\Omega_{2}$ является существенным: при $\epsilon$, фиксированным нашим выбором $H_{2}$, член $\epsilon^{2} \Omega_{2}$ дает поправку к частоте периодического солитона при удалении от точки $H=H_{0}$.

Поступая подобным образом с высшими степенями $\epsilon$, мы можем построить гомоклиническое решение $u(x), v(x), \mathcal{A}(x), \mathcal{B}(x)$ до любого порядка; одновременно мы получим коэффициенты при всех степенях $\epsilon$ в разложении $\Omega$.

\section{4. УДВОЕНИЕ ПЕРИОДА В РАМКАХ РЕДУЦИРОВАННЫХ УРАВНЕНИЙ}

Покажем, как можно отследить бифуркации удвоения периода в рамках приближения с небольшим числом мод. Полезным наблюдением здесь оказывается тот факт, что редуцированная система с $N$ гармониками и основной частотой $\Omega$ может быть переформулирована как подобная редуцированная система, но уже с $N^{\prime}=2 N$ гармониками и основной частотой $\Omega^{\prime}=\Omega / 2$, где амплитуды всех нечетных гармоник полагаются равными нулю. Рассмотрим, например, редуцированную систему, учитывающую нулевую, первую и вторую гармоники, т. е. систему, получающуюся, если положить

$$
\begin{aligned}
& U(x, t)=u(x)+\mathcal{P}(x) e^{i \Omega t}+\text { к. с. }+\mathcal{A}(x) e^{2 i \Omega t}+\text { к. с. }, \\
& V(x, t)=v(x)+\mathcal{Q}(x) e^{i \Omega t}+\text { к. с. }+\mathcal{B}(x) e^{2 i \Omega t}+\text { к. с. }
\end{aligned}
$$


Здесь $u$ и $v$ - вещественные функции, а $\mathcal{A}, \mathcal{B}, \mathcal{P}$ и $\mathcal{Q}$ - комплексные коэффициентные функции, стремящиеся к нулю при $|x| \rightarrow \infty$. Эта редуцированная система, соответствующая $N=2$, записывается в виде

$$
\begin{aligned}
u_{x x}- & +2 u\left[u^{2}+v^{2}+6\left(|\mathcal{P}|^{2}+|\mathcal{A}|^{2}\right)+2\left(|\mathcal{Q}|^{2}+|\mathcal{B}|^{2}\right)\right]+ \\
& +4 v\left(\mathcal{P} \mathcal{Q}^{*}+\mathcal{P}^{*} \mathcal{Q}+\mathcal{A} \mathcal{B}^{*}+\mathcal{A}^{*} \mathcal{B}\right)+6\left[\mathcal{P}^{2} \mathcal{A}^{*}+\left(\mathcal{P}^{*}\right)^{2} \mathcal{A}\right]+ \\
& +2\left[\mathcal{Q}^{2} \mathcal{A}^{*}+\left(\mathcal{Q}^{*}\right)^{2} \mathcal{A}\right]+4\left(\mathcal{P} \mathcal{Q} \mathcal{B}^{*}+\mathcal{P}^{*} \mathcal{Q}^{*} \mathcal{B}\right)-2 \Gamma v=0 \\
v_{x x}- & +2 v\left[u^{2}+v^{2}+2\left(|\mathcal{P}|^{2}+|\mathcal{A}|^{2}\right)+6\left(|\mathcal{Q}|^{2}+|\mathcal{B}|^{2}\right)\right]+ \\
& +4 u\left(\mathcal{P}^{*} \mathcal{Q}+\mathcal{Q}^{*} \mathcal{P}+\mathcal{B} \mathcal{A}^{*}+\mathcal{B}^{*} \mathcal{A}\right)+6\left[\mathcal{Q}^{2} \mathcal{B}^{*}+\left(\mathcal{Q}^{*}\right)^{2} \mathcal{B}\right]+ \\
& +2\left[\mathcal{B}\left(\mathcal{P}^{*}\right)^{2}+\mathcal{B}^{*} \mathcal{P}^{2}\right]+4\left(\mathcal{P} \mathcal{A}^{*}+\mathcal{P}^{*} \mathcal{Q}^{*} \mathcal{A}\right)+2 H v=0 \\
\mathcal{P}_{x x}- & \mathcal{P}+2 \mathcal{P}\left(3 u^{2}+v^{2}+3|\mathcal{P}|^{2}+2|\mathcal{Q}|^{2}+6|\mathcal{A}|^{2}+2|\mathcal{B}|^{2}\right)+ \\
& +4 \mathcal{Q}\left(\mathcal{A B} \mathcal{B}^{*}+\mathcal{A}^{*} \mathcal{B}+u v\right)+2 \mathcal{P} *\left(\mathcal{Q}^{2}+2 v \mathcal{B}+6 u \mathcal{A}\right)+ \\
& +4 \mathcal{Q}(v \mathcal{A}+u \mathcal{B})-i \Omega \mathcal{Q}-2 \Gamma \mathcal{Q}=0 \\
\mathcal{Q}_{x x}- & \mathcal{Q}+2 \mathcal{Q}\left(u^{2}+3 v^{2}+2|\mathcal{P}|^{2}+3|\mathcal{Q}|^{2}+2|\mathcal{A}|^{2}+6|\mathcal{B}|^{2}\right)+ \\
& +4 \mathcal{P}\left(\mathcal{B}^{*} \mathcal{A}+\mathcal{B} \mathcal{A}^{*}+u v\right)+2 \mathcal{Q}\left(\mathcal{P}^{2}+2 u \mathcal{A}+6 v \mathcal{B}\right)+ \\
& +4 \mathcal{P}^{*}(v \mathcal{A}+u \mathcal{B})+i \Omega \mathcal{P}+2 H \mathcal{Q}=0 \\
\mathcal{A}_{x x}- & \mathcal{A}+2 \mathcal{A}\left(3 u^{2}+v^{2}+6|\mathcal{P}|^{2}+2|\mathcal{Q}|^{2}+3|\mathcal{A}|^{2}+2|\mathcal{B}|^{2}\right)+2 \mathcal{A}^{*} \mathcal{B}^{2}+ \\
& +4 \mathcal{B}\left(u v+\mathcal{P} \mathcal{Q}^{*}+\mathcal{P}^{*} \mathcal{Q}\right)+2 u\left(3 \mathcal{P}^{2}+\mathcal{Q}^{2}\right)+4 v \mathcal{P} \mathcal{Q}-2 i \Omega \mathcal{B}-2 \Gamma \mathcal{B}=0 \\
\mathcal{B}_{x x}- & \mathcal{B}+2 \mathcal{B}\left(u^{2}+3 v^{2}+2|\mathcal{P}|^{2}+6|\mathcal{Q}|^{2}+2|\mathcal{A}|^{2}+3|\mathcal{B}|^{2}\right)+2 \mathcal{B}^{*} \mathcal{A}^{2}+ \\
& +4 \mathcal{A}\left(u v+\mathcal{P} \mathcal{Q}^{*}+\mathcal{P} * \mathcal{Q}\right)+2 v\left(\mathcal{P}^{2}+3 \mathcal{Q}^{2}\right)+4 u \mathcal{P} \mathcal{Q}+2 i \Omega \mathcal{A}+2 H \mathcal{B}=0 .
\end{aligned}
$$

Если положить $\mathcal{P}=\mathcal{Q}=0$, то данная система сведется к системе $(7)$, где вместо $\Omega$ будет стоять $2 \Omega$. Следовательно, любое решение уравнений (7) является решением уравнений (13), и это решение является “тривиальным" в том смысле, что оно имеет $\mathcal{P}=\mathcal{Q}=0$.

Решение с $\mathcal{P}, \mathcal{Q} \neq 0$ может отщепляться от тривиального решения. В точке такой бифуркации (типа "вилка") оператор линеаризации вблизи тривиального решения имеет нулевое собственное значение. Этот оператор является матрицей размеpa $(10 \times 10)$, диагональные элементы которой - дифференциальные операторы второго порядка, а недиагональные - произведения степеней функций $u, v, \mathcal{A}$ и $\mathcal{B}$ (важно, что не присутствуют степени функций $\mathcal{P}$ и $\mathcal{Q})$. Этот матрично-дифференциальный оператор действует на столбцы вида $\left(\vec{X}^{(6)}, \vec{Y}^{(4)}\right)^{\mathrm{T}}$, где

$$
\vec{X}^{(6)}=(\delta u, \delta v, \operatorname{Re} \delta \mathcal{A}, \operatorname{Im} \delta \mathcal{A}, \operatorname{Re} \delta \mathcal{B}, \operatorname{Im} \delta \mathcal{B})^{\mathrm{T}}, \quad \vec{Y}^{(4)}=(\operatorname{Re} \delta \mathcal{P}, \operatorname{Im} \delta \mathcal{P}, \operatorname{Re} \delta \mathcal{Q}, \operatorname{Im} \delta \mathcal{Q})^{\mathrm{T}} .
$$

Легко убедиться в том, что матрица размера $(10 \times 10)$ является блочно-диагональной и имеет ненулевой блок размера $(6 \times 6)$ в верхнем левом углу и ненулевой блок размера $(4 \times 4)$ в нижнем правом углу. При этом блок размера $(6 \times 6)$ является оператором линеаризации системы (7); его нулевые собственные значения сигнализируют о возникновении у тривиального решения бифуркаций типа "седло-узел". Что касается удвоения периода периодических решений, аппроксимируемых системой (7), то о них сигнализируют нулевые собственные значения блока размера $(4 \times 4)$.

Следовательно, все, что требуется, для того чтобы обнаружить бифуркацию удвоения периода с помощью редуцированной системы (7) с $N=1$, - это следить за 
собственными значениями матрично-дифференциального оператора размера $(4 \times 4)$

$$
\left(\begin{array}{cccc}
\mathcal{L}_{1}+m_{r} & m_{i} & 2 \Gamma+r+s_{r} & s_{i}-\Omega \\
m_{i} & \mathcal{L}_{1}-m_{r} & s_{i}+\Omega & 2 \Gamma+r-s_{r} \\
r+s_{r} & s_{i}+\Omega & \mathcal{L}_{0}+n_{r} & n_{i} \\
s_{i}-\Omega & r-s_{r} & n_{i} & \mathcal{L}_{0}-n_{r}
\end{array}\right)
$$

где

$$
\begin{gathered}
\mathcal{L}_{0}=-\frac{d^{2}}{d x^{2}}+1-2 u^{2}-6 v^{2}-4|\mathcal{A}|^{2}-12|\mathcal{B}|^{2}-2 H, \\
\mathcal{L}_{1}=-\frac{d^{2}}{d x^{2}}+1-6 u^{2}-2 v^{2}-12|\mathcal{A}|^{2}-4|\mathcal{B}|^{2}, \\
m_{r}+i m_{i}=4(v \mathcal{B}+3 u \mathcal{A}), \quad n_{r}+i n_{i}=4(u \mathcal{A}+3 v \mathcal{B}), \quad s_{r}+i s_{i}=-4(v \mathcal{A}+u \mathcal{B})
\end{gathered}
$$

и $r=-4\left(u v+\mathcal{A B}^{*}+\mathcal{B A}^{*}\right)$. Подобным образом бифуркации удвоения периода могут быть обнаружены при использовании редуцированной системы с $N \geqslant 2$.

\section{5. ПРОДОЛЖЕНИЕ РЕШЕНИЙ РЕДУЦИРОВАННЫХ УРАВНЕНИЙ}

Предлагаемый приближенный метод на основе небольшого числа мод был опробован на примере расчета бризерного решения уравнения (1), которое возникает в результате бифуркации Хопфа солитона $\psi_{+}$. Периодическое по времени решение полного уравнения в частных производных (1) может быть продолжено (прослежено по траектории) по параметру $h$ при фиксированном значении $\gamma$. При $\gamma>0.27$ кривая продолжения заканчивается на другом стационарном решении, а именно на связанном состоянии солитона $\psi_{+}$и двух солитонов $\psi_{-}$[9]. Типичная бифуркационная диаграмма изображена сплошной линией на рисунке, где период бризера представлен как функция интенсивности накачки). Верхняя ветвь этой кривой соответствует решению, которое можно грубо описать как "периодическое односолитонное решение", тогда как нижнюю ветвь можно описать как "периодический триплет". Эта классификация является довольно грубой, поскольку три горба у решения появляются еще на верхней ветви, до того как мы достигнем точки поворота.

В нашем приближенном анализе мы ограничились конечной суммой с $N=2$; это второе по грубости приближение после $N=1$. Для сравнения с решением полного уравнения в частных производных (1) мы продолжили решение редуцированной системы (13) по параметру $h$ при фиксированном значении $\gamma(\gamma>0.27)$. Начальной точкой продолжения был взят аналог солитона $\psi_{+}$в редуцированной системе: $u=\operatorname{sech} x, v=\mathcal{A}=\mathcal{B}=\mathcal{P}=\mathcal{Q}=0$. Во всех рассмотренных нами случаях указанное приближение тремя гармониками достаточно точно воспроизводило общий вид бифуркационной кривой.

Типичный результат численного расчета решения показан на рисунке штриховой кривой. Как и сплошная линия, соответствующая полному уравнению в частных производных (1), штриховая кривая имеет точку поворота и заканчивается решением с тремя горбами (редуцированным аналогом стационарного связанного состояния $\left.\psi_{(-+-)}\right)$. Как и ожидалось, две кривые совпадают вблизи начальной и конечной точек - там, где решение близко к стационарному. Действительно, поскольку 


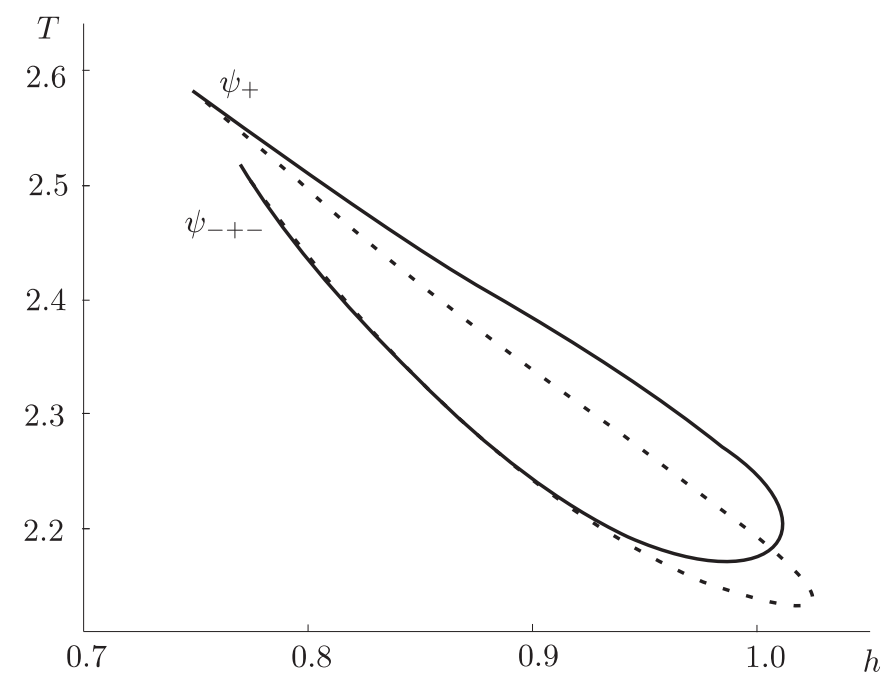

Период бризера как функция амплитуды накачки при фиксированном коэффициенте диссипации. Сплошная кривая - решение уравнения в частных производных (1). Штриховая кривая - приближение, полученное с помощью численного решения системы (13). Здесь $\gamma=0.35$.

$\mathcal{P}, \mathcal{Q}, \mathcal{A}$ и $\mathcal{B}$ в этих областях невелики, первые два уравнения в системе (13) совпадают со стационарной частью уравнений (5). В меньшей степени можно было ожидать, что точная и приближенная кривые совпадут на большей части одной из их двух составляющих их ветвей за пределами малой окрестности конечной точки. Объяснение этой неожиданной точности заключается в малости амплитуды первой гармоники (и, следовательно, всех гармоник более высокого порядка) на большей части трехсолитонной ветви (солитоны $\psi_{-}$, связанные в комплекс $\psi_{-+-}$, обнаруживают тенденцию гасить осцилляции солитона $\left.\psi_{+}\right)$.

\section{6. ЗАКЛЮЧИТЕЛЬНЫЕ ЗАМЕЧАНИЯ}

Суть нашего приближенного метода состоит в сведении двумерной граничной задачи к одномерной. В то время как решение граничной задачи для уравнения в частных производных может потребовать значительных затрат компьютерных ресурсов, а при их отсутствии быть недостаточно точным, процедура решения редуцированной системы обыкновенных дифференциальных уравнений является быстрой, а само решение может быть получено с любой наперед заданной точностью. Редуцированная система позволяет не только определить область существования бризеров, но также исследовать их устойчивость и бифуркации. Недостаток метода заключается в том, что если амплитуда колебаний во времени недостаточно мала, то редуцированная система с малым значением $N$ (таким, как $N=2$ ) может давать слишком грубое приближение решения уравнения в частных производных. Однако этот недостаток можно преодолеть просто с помощью увеличения $N$, не рискуя при этом превысить доступные вычислительные ресурсы. 
Благодарности. Мы благодарим Игоря Барашенкова и Юрия Гайдидея за полезные советы. Работа Н. В. Алексеевой была поддержана National Research Foundation of South Africa (грант № 67982). Работа Е.В. Земляной была поддержана Department of Science and Technology of South Africa в рамках Программы сотрудничества ОИЯИ с научными центрами ЮАР и частично поддержана РФФИ (грант № 09-01-00770).

\section{Список литературы}

[1] J. W. Miles, J. Fluid Mech., 148 (1984), 451-460; C. Elphick, E. Meron, Phys. Rev. A, 40:6 (1989), 3226-3229; W. Zhang, J. Viñals, Phys. Rev. Lett., 74:5 (1995), 690-693, arXiv: patt-sol/9311005; S. P. Decent, A. D. D. Craik, J. Fluid Mech., 293 (1995), 237-268; S. P. Decent, Fluid Dynam. Res., 21:2 (1997), 115-137; L. Zhang, X. Wang, Z. Tao, Phys. Rev. E, 75:3 (2007), 036602, 6 pp.

[2] I. H. Deutsch, I. Abram, J. Opt. Soc. Amer. B, 11:11 (1994), 2303-2313; A. Mecozzi, W. L. Kath, P. Kumar, C. G. Goedde, Opt. Lett., 19:24 (1994), 2050-2052; S. Longhi, Opt. Lett., 20:7 (1995), 695-697; V. J. Sánchez-Morcillo, I. Pérez-Arjona, F. Silva, G. J. de Valcárcel, E. Roldán, Opt. Lett., 25:13 (2000), 957-959.

[3] I. V. Barashenkov, M. M. Bogdan, V. I. Korobov, Europhys. Lett., 15:2 (1991), 113.

[4] B. Denardo, B. Galvin, A. Greenfield, A. Larraza, S. Putterman, W. Wright, Phys. Rev. Lett., 68:11 (1992), 1730-1733; W.-Z. Chen, Phys. Rev. B, 49:21 (1994), 15063-15066; G. Huang, S.-Y. Lou, M. G. Velarde, Internat. J. Bifur. Chaos Appl. Sci. Engrg., 6:10 (1996), 1775-1787; I. V. Barashenkov, N. V. Alexeeva, E. V. Zemlyanaya, Phys. Rev. Lett., 89:10 (2002), 104101, 4 pp., arXiv: nlin/0112047.

[5] M. Bondila, I. V. Barashenkov, M. M. Bogdan, Physica D, 87:1-4 (1995), 314-320.

[6] N. V. Alexeeva, I. V. Barashenkov, D. E. Pelinovsky, Nonlinearity, 12:1 (1999), 103-140.

[7] Е. В. Земляная, Н. В. Алексеева, ТМФ, 159:3 (2009), 536-544.

[8] D. M. Ambrose, J. Wilkening, Comm. Appl. Math. Comput. Sci., 4:1 (2009), 177-215, arXiv: 0811.4205; J. Nonlinear Sci., 20:3 (2010), 277-308, arXiv: 0804.3623.

[9] I. V. Barashenkov, E.V. Zemlyanaya, T. C. van Heerden, Time-periodic solitons in a damped-driven nonlinear Schrödinger equation, Phys. Rev. E (to appear), arXiv: 1103.3604. 